[2] Orlando A, Orlando R, Ciccia F, et al. Ann Rheum Dis 2017 Sep;76(9): e31.

Disclosure of Interest: None declared

DOI: 10.1136/annrheumdis-2018-eular.3068

WEDNESDAY, 13 JUNE 2018: RA therapy - new molecules and new strategies

\section{OP0030 CORTICOSTEROID BRIDGING STRATEGIES WITH METHOTREXATE MONOTHERAPY IN EARLY RHEUMATOID AND UNDIFFERENTIATED ARTHRITIS; A COMPARISON OF EFFICACY AND TOXICITY IN THE TREACH AND IMPROVED STUDIES}

J.A. van der Pol ${ }^{1}$, E.G. Brilman ${ }^{1}$, P.H. de Jong ${ }^{2,3}$, A.E. Weel $^{2,3}$, J.M. Hazes ${ }^{3}$, T. W. Huizinga ${ }^{1}$, C.F. Allaart ${ }^{1}{ }^{1}$ LUMC, Leiden; ${ }^{2}$ Maasstad Hospital; ${ }^{3}$ Erasmus $M C$, Rotterdam, Netherlands

Background: What is the optimal glucocorticoid (GC) bridging therapy with MTX monotherapy in early arthritis?

Objectives: To compare short term clinical efficacy of high and low dose GC tapering schedules with MTX monotherapy in 2 clinical trials in early rheumatoid arthritis (RA) and undifferentiated arthritis (UA) patients.

Methods: In tREACH, early RA and UA (arthritis in $\geq 1$ joint(s), $<1$ year symptoms) patients were randomised to 3 different treatment arms. For this analysis we only use the data of arm C: oral GCs (prednisone) (15 mg/day, tapered to 0 in 10 weeks) with MTX monotherapy ( $25 \mathrm{mg} /$ week); low dose GC tapering schedule (LDGC).

In IMPROVED RA and UA (arthritis in $\geq 1$ joint and $\geq 1$ other painful joint, $<2$ years symptoms) patients were treated with prednisone $(60 \mathrm{mg} /$ day, tapered in 7 weeks to $7.5 \mathrm{mg} / \mathrm{day}$, continued to 4 months)+MTX monotherapy (25 mg/week); high dose GC tapering schedule (HDGC). We compared\%DAS-remission $(<1.6)$ and low disease activity $(\leq 2.4)$ at first evaluation (3 months tREACH, 4 months IMPROVED) and DAS and HAQ over time. After multivariate normal imputation we applied generalised estimating equations (GEE) for linear outcomes and logistic regression models for binary outcomes, adjusted for potential baseline confounders (figure 1). Adverse events were compared between treatment arms using $\chi 2$-square tests.

Results: Patients with a HDGC ( $n=610)$ had shorter symptom duration and higher HAQ, were less often seropositive (ACPA positive $56.0 \%$ vs $77.3 \%$, RF positive $58.1 \%$ vs $65 \%)$ and more often had UA (20.3\% vs $2.1 \%)$ than patients with a LDGC ( $n=97)$. Baseline DAS was comparable.

At the first evaluation time point (median 3.06 (IQR 2.99-3.22) months in LDGC, 4.01. (3.8-4.17) in HDGC) DAS and HAQ had decreased significantly less after 3 months LDGC: DAS $\beta(95 \% \mathrm{Cl}) 0.500(0.276 ; 0.725)$, and HAQ 0.330 (0.189; 0.470 ) than after 4 months HDGC (figure 1).

Compared to the HDGC patients, patients with the LDGC had a significantly lower chance of achieving DAS-remission $63.4 \%$ vs $28.9 \%$ (OR (95\% Cl) $0.215(0.124$; $0.373)$ and low disease activity $80.6 \%$ vs $55.7 \%$ ((OR ( $95 \% \mathrm{Cl}) 0.249$ (0.143; $0.435)$ ). Presence of ACPA was positively associated with achieving DAS-remission in the HDGC group, but not in the LDGC group. Per 100 patient years, 7.98 serious adverse events were reported in the HDGC and 23.4 in the LDGC $(p=0.004)$. Hypertension, hyperglycemia $(>7.8 \mathrm{mmol} / \mathrm{L})$, gastrointestinal complaints and liverenzymes above normal were reported in similar frequencies across all groups. In patients with a LDGC more headaches, skin rashes, creatinine above normal range and any decrease in haematology blood counts were reported (data not shown).
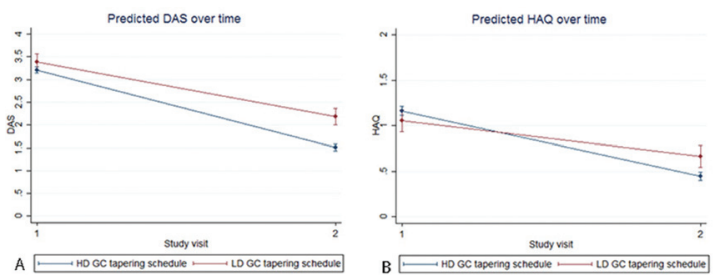

Abstract OP0030 - Figure $1 \mathrm{~A}$ : Predicted DAS over time, B: PRedicted HAQ over time. All predictions are from multiple imputed models, adjusted for age, gender body mass index, presence of ACPA, presence of rheumatoid factor, symptom duration, effect over time (in GEE) and baseline DAS (for binary out-comes). DAS, Disease Activity Score; HAQ Health Assessment Questionnarie; HD GC, high dose glucocorticoids, LD GC, low dose glucocorticoids
Conclusions: In early arthritis patients, GC bridging therapy with prednisone $60 \mathrm{mg}$ daily tapered in 7 weeks to and continued at $7.5 \mathrm{mg}$ daily in combination with MTX monotherapy was associated with better clinical outcomes and without additional effects than prednisone $15 \mathrm{mg}$ daily tapered to nil in 10 weeks in combination with MTX monotherapy, after correction for baseline age, gender, DAS, body mass index, presence of ACPA, presence of rheumatoid factor, symptom duration, and (in GEE) time from baseline.

Disclosure of Interest: J. van der Pol: None declared, E. Brilman: None declared, P. de Jong: None declared, A. Weel Grant/research support from: tREACH study was supported by an unrestricted grant from Pfizer bv. [0881102 217]. Pfizer had no involvement in study design; in collection, analysis and interpretation of data; writing of the report and decision to submit for publication. J. Hazes: None declared, T. Huizinga: None declared, C. Allaart Grant/research support from: The work was supported by AbbVie during the first year of the IMPROVED study. Study design, data collection, trial management, data analysis and preparation of the manuscript were performed by the authors. DOI: 10.1136/annrheumdis-2018-eular.1912

\section{\begin{tabular}{|l|l}
\hline OP0031 THE IMPORTANCE OF ASSESSING MULTIPLICATIVE \\
\hline
\end{tabular} AND ADDITIVE INTERACTION: EXAMINING THE EFFECT OF GLUCOCORTICOID THERAPY ON MORTALITY IN PATIENTS WITH RHEUMATOID ARTHRITISAND CONCOMITANT TYPE II DIABETES}

R. Costello ${ }^{1}$, A. Marsden ${ }^{2}$, M. Movahedi ${ }^{3}$, M. Lunt ${ }^{1}$, R. Emsley ${ }^{2}$, W.G. Dixon ${ }^{1}$. ${ }^{1}$ Arthritis Research UK Centre for Epidemiology; ${ }^{2}$ Centre for biostatistics, The University of Manchester, Manchester, UK; ${ }^{3}$ Ontario Best Practices Research Initiative, University Health Network, Toronto, Canada

Background: Glucocorticoids (GC) are widely used to treat rheumatoid arthritis (RA), however they are known to have risks associated with them. It has been shown that GCs increase the risk of diabetes mellitus (DM). A few studies have investigated the long-term effects of GC use on outcomes in DM, but not in RA specifically. As people with RA already have increased risk of cardiovascular (CV) disease, the additional burden of DM and GCs may be important. If the effect of GCs was dependent on DM we would say there is effect modification and this can be on the additive scale, corresponding to variation in the absolute treatment effect, e.g. the risk difference (RD), across DM status, or the multiplicative scale, corresponding to variation in the relative treatment effect e.g. the rate ratio $(R R){ }^{1}$ Objectives: To examine in patients with RA 1) whether all-cause and CV mortality rates differ by GC and DM status, and 2) whether DM modifies the relationship between GC and all-cause and CV mortality on multiplicative and additive scales. Methods: Patients with RA and linkage to mortality data were identified from the Clinical Practice Research Datalink ( $n=9085)$, a database of primary care electronic medical records in the UK. RR and RD for ever GC use were calculated by DM status. Cox proportional hazards $(\mathrm{PH})$ regression models were fitted with an interaction term for DM and use of GC to assess multiplicative interaction. Additive interaction was measured with the Relative Excess Risk due to Interaction $(\mathrm{RERI})^{2}$ where a value different from zero indicates a difference in the absolute effect of treatment.

Results: Those with DM and ever treated with GCs had a 3-fold increased allcause mortality RR $(95 \% \mathrm{Cl}: 2.27,4.09)$ whilst those without DM had a slightly higher RR $(3.46(95 \% \mathrm{Cl}: 2.95,4.07))$. However those with DM had a higher RD: 36.46 deaths per 1000 patient years (pyrs) $(95 \% \mathrm{Cl}: 27.5,45.41)$ compared to those without DM: RD 22.83 deaths per 1000 pyrs $(95 \% \mathrm{Cl}: 19.83,25.82)$ because of higher baseline mortality rates. A similar pattern was seen for $\mathrm{CV}$ mor tality. The adjusted Cox PH model for all-cause mortality showed no evidence of multiplicative interaction, but there was significant additive interaction (RERI 0.86 (95\% Cl: 0.18, 1.54)). For CV mortality there was no interaction on either scale. Conclusions: Methodologically, this study showed assessing interaction on the additive and multiplicative scales can lead to different conclusions and should be considered carefully. In this study significant interaction was seen on additive scale but not on the multiplicative scale due to higher baseline rates in patients with DM. Clinically, this study provides some evidence that long-term GC therapy may be particularly harmful in patients with RA and DM.

\section{REFERENCES}

[1] VanderWeele TJ, Knol MJ. A tutorial on interaction. Epidemiologic Methods $2014 ; 3(1): 33-72$

[2] Li R, Chambless L. Test for additive interaction in proportional hazards models. Annals of epidemiology 2007 Mar;17(3):227-236.

Disclosure of Interest: None declared

DOI: 10.1136/annrheumdis-2018-eular.1755 